\title{
Parietal cortex and a spatial cognitive map
}

\author{
RAYMOND P. KESNER \\ University of Utah, Salt Lake City, Utah \\ and \\ JEFFREY M. LONG \\ Gerontology Research Center, National Institute on Aging, \\ National Institutes of Health, Baltimore, Maryland
}

\begin{abstract}
A framework is proposed, based on the assumption that the posterior parietal cortex stores longterm memory information in the form of a spatial cognitive map, reflecting the operation of perceptual representations of relations among spatial features and landmarks as well as the operation of attentional processes to bind spatial features and landmarks and to selectively attend to the critical features that form spatial cognitive maps. Evidence in support of this assumption, based on an analysis of specific properties of neural systems that store long-term memory information, is presented.
\end{abstract}

The purpose of this short overview is to develop the idea that the posterior parietal cortex (PPC) stores long-term memory for spatial information in the form of a spatial cognitive map. In past research using the rat model, difficulties have emerged in analyzing the exact contribution of the PPC in mediating complex spatial information, because different investigators have destroyed different anatomical regions that were presumed to alter the function of the PPC. However, in recent years there has been some general agreement regarding the area that delineates the PPC in the rat. As described in this issue by McDaniel, Williams, Attaway, and Compton (1998) and Corwin and Reep (1998), and in a previous study by Kolb and Walkey (1987), the new anatomical findings have resulted in a definition of the rodent PPC as cortical tissue that has pronounced connections with the lateral posterior thalamus (LP), lateral dorsal thalamus (LD), and posterior ( $\mathrm{Po}$ ) nuclei, but no input from the ventrobasal complex (VB) or dorsal lateral geniculate (DLG) (Reep, Chandler, King, \& Corwin, 1994). It should be noted that rats have no true pulvinar, but it is likely that the homologous structure is area LP. With these criteria, the PPC region of the rat is approximately 3.5$4.5 \mathrm{~mm}$ caudal to bregma, and $1.5-5 \mathrm{~mm}$ lateral to midline (Reep et al., 1994). This region of rodent cortex has connections with the ventrolateral orbital cortex (VLO) and medial orbital cortex (MO), medial agranular cortex $(\mathrm{AGm})$, and retrosplenial cortex. These patterns of thalamo-cortical and cortico-cortical connections are similar to those in humans and nonhuman primates, and there now seems to be general agreement among investigators of this anatomical definition of rat PPC.

There is also agreement of the general type of processing functions carried out by the rodent PPC. In gen-

Correspondence should be addressed to R. P. Kesner, Department of Psychology, University of Utah, Salt Lake City, UT 84112 (e-mail: rpkesner@behsci.utah.edu). eral, the authors represented in the present special issue would most likely agree that the PPC is involved in spatial navigation, attention (perhaps only spatial), and memory for body (or head) representations. Beyond these generalizations, however, each investigator has a slightly different way of conceptualizing the function of the PPC. Save, Poucet, Foreman, and Thinus-Blanc (1998) view the PPC as the interface between egocentric and allocentric coding of space; the PPC is thought to be a critical substrate that is involved in associating egocentric information (visuospatial and internal movement cues) and transferring this information into an allocentric spatial framework. For Corwin and Reep (1998), the PPC is part of a cortical network for directed attention to multimodal information, including spatial orientation. Both McDaniel et al. (1998) and Kolb and Cioe (1998) agree that the PPC plays an important role in spatial navigation as assessed in the water maze; McDaniel, however, does not view the PPC as the permanent store of the spatial cognitive map.

Can all of the functions of PPC be put together into a single theoretical framework? We propose a framework in which the PPC stores long-term memory information in the form of a spatial cognitive map, reflecting the operation of perceptual representations of relations among spatial features and landmarks as well as the operation of attentional processes to bind spatial features and landmarks and to selectively attend to the critical features that form spatial cognitive maps. Evidence in support of this idea is based on an analysis of specific properties of neural systems that store long-term memory information which include attentional and perceptual processes.

\section{Attention}

One form of attention involves the binding of a number of important spatial as well nonspatial features to constitute an abstract representation of space in the form of a spatial cognitive map. If the PPC directly supports the bind- 
ing of multiple features through attentional processes, it should be the case that the PPC is not involved in memory for individual features (i.e., subunits that make up the map), but that the PPC is involved when these features must be combined, perhaps through attention, to form more complex representations of the environment. A brief outline of the evidence supporting this idea is presented below.

There are no impairments in the discrimination of or short-term memory for single spatial features, including spatial location and allocentric and egocentric spatial distance (Long \& Kesner, 1996; Long \& Kesner, in press). Similarly, there are no impairments in discriminating between visual objects in terms of either new learning or performance of a previously learned visual discrimination (Davis \& McDaniel, 1993; Kolb, Buhrmann, McDonald, \& Sutherland 1994; Long, Mellem, \& Kesner, 1998). However, some early studies did report deficits on visual discrimination tasks (Boyd \& Thomas, 1977; McDaniel \& Wall, 1988). Different stimuli, testing procedures, and lesion sites most likely account for the conflicting data. In general, it appears that rats with lesions of the PPC are not impaired in simple discrimination tasks. An initial report of PPC lesions impairing performance in a task in which the rat must respond differently to the presence of a white or black cue card was found to be reversed by increasing the negative consequence for errors (Davis \& McDaniel, 1993, vs. McDaniel \& Skeel, 1993). When the task is more complex, involving the association of objects and places (components of a spatial cognitive map), then the parietal cortex plays an important role. Support for this comes from the finding that rats with parietal lesions are impaired in the acquisition and retention of a spatial location plus object discrimination (paired associate task), but show no deficits for only spatial or object discriminations (Long \& Kesner, 1995; Long et al., 1998). Comparable deficits are found within an egocentric-allocentric distance paired-associate task, but no deficit for an object-object paired-associate task, suggesting that spatial features are essential in activating and involving the parietal cortex (Kesner, unpublished observations).

Also, rats with parietal cortex lesions are clearly impaired in learning in mazes (Thomas \& Weir, 1975). For example, PPC lesions impair acquisition but not retention in a 14-unit T-maze (Jucker, Kametani, Bresnahan, \& Ingram, 1990; Spangler et al., 1994). Furthermore, rats with parietal cortex lesions display deficits in both the acquisition and the retention of spatial navigation tasks that are presumed to measure the operation of a spatial cognitive map within a complex environment (DiMattia \& Kesner, 1988b; Kesner, Farnsworth, \& Kametani, 1992). They also display deficits in the acquisition and retention of spatial recognition memory for a list of five spatial locations (DiMattia \& Kesner, 1988a). In a complex discrimination task in which a rat has to detect the change in location of an object in a scene, rats with parietal cortex lesions are profoundly impaired
(DeCoteau \& Kesner, 1998). Similarly, in a reaction-tochange task with a complex array of objects, rats with parietal lesions do not react to a spatial change or a change consisting of removal of a stimulus (Save, Poucet, Foreman, \& Buhot, 1992).

There is some additional support to suggest that the parietal cortex may be a site for long-term representation of complex spatial information. Cho and Kesner (1996) and Cho, Kesner, and Brodale (1995) have shown that rats with parietal cortex lesions have a nongraded retrograde amnesia for four, but not two, previously learned spatial discriminations prior to surgery, suggesting that the deficit cannot be due to a performance or anterograde amnesia problem, but appears rather to be a function of the number or complexity of the spatial information to be stored and to be remembered.

Finally, it should be noted that in rats, neurons have been found within the parietal cortex that encode spatial location and head direction information and that many of these cells are sensitive to multiple cues, including visual, proprioceptive, sensorimotor, and vestibular cue information (Chen, Lin, Barnes, \& McNaughton, 1994; Chen, Lin, Green, Barnes, \& McNaughton, 1994; McNaughton, Chen, \& Marcus, 1991). For example, as nicely summarized by Chen and Nakamura (1998), singleunit recording data suggest that rat parietal cortex may be involved in body orientation representations and spatial memories. A small percentage of cells in the PPC respond selectively to the rat's head orientation (Chen, Lin, Barnes, \& McNaughton, 1994, Chen, Lin, Green, et al., 1994). These head direction cells persist after the removal of visual cues (either by physically removing the cues or turning off the lights), and a subset associate angular motion with head orientation (Chen, Lin, Barnes, \& McNaughton, 1994; Chen, Lin, Green, et al., 1994). Therefore, it appears that parietal cortex cells are responsive to an interaction between visual and sensorimotor inputs. Neurons have also been found in the parietal cortex that encode spatial location information (Chen, Lin, Barnes, \& McNaughton, 1994; Chen, Lin, Green, et al., 1994; McNaughton et al., 1994; Nakamura \& Takarajima, 1996). Some of these PPC cells maintain mnemonic information for head direction (Chen, Lin, Barnes, \& McNaughton, 1994; Chen, Lin, Green, et al., 1994) and the spatial location of a tone (Nakamura \& Takarajima, 1996) .

In humans, there is often a deficit associated with spatial aspects of the patients' environments. These include an inability to draw maps or diagrams of familiar spatial locations, to use information to guide them in novel or familiar routes, to discriminate near from far objects, and to solve complex mazes. There is a general loss of "topographic sense," which may involve loss of long-term geographical knowledge as well as an inability to form cognitive maps of new environments. With PET scan and functional MRI data, it can be shown that complex spatial information results in activation of the parietal cortex (Ungerleider, 1995). Thus, memory for complex spa- 
tial information appears to be impaired (Benton, 1969; DeRenzi, 1982). In addition, (Friedman-Hill, Robertson, \& Treisman, 1995) have reported a patient with symmetrical bilateral parieto-occipital damage who can discriminate shapes and letters, but cannot correctly "bind" the color and size of two or more shapes. The subject experiences illusory conjunctions (ICs) in which the memory for the color/shape combination is incorrect. This provides an example in which the PPC is shown to process the combination of two or more features. Again, the processing of the individual features is unimpaired; it is the process of combining specific features that appears faulty.

The preceding evidence suggests that the PPC is not involved in the processing of single features, such as in the simple discrimination of objects, colors, or distances. However, when these individual features must be combined to form more complex spatial representations, then the PPC is recruited.

It should be noted that the parietal cortex is probably not the only neural region that mediates long-term memory for spatial information. For example, topographical amnesia has also been reported for patients with parahippocampal lesions, and spatial navigation deficits have also been found following retrosplenial and entorhinal cortex lesions (Habib \& Sirigu, 1987; Sutherland, Whishaw, $\&$ Kolb, 1988). Thus, other neural regions (e.g., the parahippocampal cortex, entorhinal cortex, and retrosplenial cortex) may also contribute to the long-term representation of a spatial cognitive map.

A different form of attention involves selectivity in directing, focusing, and switching attention from one focus to another. The failure to orient, respond, or report to novel or meaningful stimuli presented to the contralateral side of the lesion is often interpreted as reflecting an inability to attend to information presented to one side of space or one side of the body. Support for an active role for the parietal cortex in supporting a selective attention process comes from the findings of neglect of spatial information observed in rats and humans (Heilman, Watson, \& Valenstein, 1993; King \& Corwin, 1993). For example, PPC lesions in rats can result in visual, auditory, and tactile neglect (Crowne, Richardson, \& Dawson, 1986; King $\&$ Corwin, 1993) and a rodent version of simultaneous extinction (Save et al., 1992). In addition, as in humans, the right-hemisphere rat PPC is involved to a greater extent in spatial processing than is the left-hemisphere PPC (Crowne, Novotny, Maier, \& Vitols, 1992; King \& Corwin, 1992).

In humans with parietal cortex lesions, there are also spatial neglect and deficits in spatial attention (Heilman et al., 1993; Rafal \& Roberston, 1995). In a well-researched paradigm, spatial covert attention is measured by the difference in reaction time (validity effect) between trials in which the cues that precede the target on the same side (valid cues) and trials in which the cue is on the opposite side (invalid cues). It can be shown that in this paradigm, patients with posterior parietal cortex lesions display an increase in the validity effect, which is thought to be due to a difficulty in disengaging attention from an invalid cue (Posner, 1980; Posner, Walker, Friedrich, \& Rafal, 1984). Analogous tests in rodents with unilateral PPC lesions have, however, not resulted in any deficits in covert spatial orienting responses; that is, there were no deficits in the ability to disengage attention (Rosner \& Mittleman, 1996; Ward \& Brown, 1997). There is a possibility that bilateral PPC lesions are necessary in order to produce covert attentional deficits in the rat.

\section{Spatial Perceptual Memory}

Schacter (1994) has suggested that one process associated with posterior neocortical systems is the operation of a perceptual representation system that can be accessed via a repetition priming procedure. This perceptual representation system can take the form of a structural description of representations of relations among spatial features and landmarks (objects), in order to specify the structure of a spatial cognitive map. It is, therefore, possible that the PPC supports a spatial-cognitive-mapbased structural perceptual system. One should then be able to measure the operation of spatial-perceptual memory by using a spatial repetition priming paradigm.

Thus, a new paradigm using a repetition spatial priming task was developed to examine the role of the PPC in perceptual spatial memory. In this task, rats were trained on a black versus white object discrimination in a dry land version of a water maze (a round space with many holes). On every trial, rats are started from a black start box and trained to find food beneath the black or white object. The two objects can appear in one of eight spatial locations on the maze, with three locations to the right, three to the left, and two straight head relative to the start box. The locations of the black and white objects were randomly varied with respect to the right and the left. During training, successive locations were not presented. Latency from opening the door until the object was displaced was measured. The animals were trained ( 16 trials per day) until they did not make any errors and latency to respond was consistent and rapid. After training, the rats received in the positive priming condition on 4 of the 16 trials an immediate repetition of the prior location. After 48 repetition trials, all rats in the positive priming condition ran more quickly to the repeated location. This facilitation manifested itself only for an immediate repetition, because if any other spatial location occurred prior to a specific location repetition, the facilitation did not occur. After training, the rats received parietal cortex or control lesions and were then retested. The results indicate that the parietal cortex, but not control lesioned rats were impaired for the positive priming condition. Thus, it appears that perceptual spatial memory as measured by positive spatial priming is mediated by the PPC.

In a second experiment, perceptual spatial memory was tested in a 12-arm maze using an implicit memory procedure. In addition, comparisons were made using an 
explicit memory procedure aimed at measuring shortterm spatial memory. A continuous recognition procedure was used to train rats on a 12-arm radial maze. Each rat was allowed to visit a sequence of 12 arms per day in an order predetermined for that trial. Of the 12 arms visited, either 3 or 4 of the arms were repeated within the running sequence. The arms selected for repetition varied according to lag (0-6) or to the number of arms that occurred between the first visit to an arm and its repetition. In order to gain access to each arm, the animal was required to orient to a cue on the Plexiglas door at the entrance of the arm. Once the animal oriented to the cue, the door was lowered, and the latency for the animal to reach the end of the arm was measured. Two groups of rats were trained, one on an implicit training procedure and one on an explicit training procedure. The implicit group received reinforcement at the end of each arm, regardless of whether the arm was a novel arm or a repeated arm. This group showed decreased latencies when visiting repeated arms. The explicit group received reinforcement only when visiting an arm for the first time in a given sequence. This group showed increased latencies for repeated arms. After training, rats received total hippocampus, parietal cortex, or sham-operated and cortical control lesions. The results indicate that, following total hippocampal ablation, the performance of the rats in the implicit condition was not significantly different from preoperative performance, whereas rats in the explicit condition showed a deficit-a significant decrease in latency to return to an arm. Following parietal lesions, the rats in the implicit condition showed a deficit-an increase in latency to return to an arm-whereas the performance of the rats in the explicit condition was not significantly different from preoperative performance. The performance of sham operated control rats and cortical control rats did not differ significantly from preoperative performance in either reinforcement condition (Chiba, Jackson-Smith, \& Kesner, 1991). Thus, a double dissociation appears to exist between the parietal cortex and hippocampus for memory operations associated with spatial perceptual memory versus memory operations associated with more elaborative processing of spatial information.

In humans, a double dissociation between implicit memory (a measure reflective of the operation of the perceptual component of long-term memory) and explicit memory (a measure reflective of the operation of shortterm recognition memory) has also been reported (Gabrieli, Fleischman, Keane, Reminger, \& Morrell, 1995; Keane, Gabrieli, Mapstone, Johnson, \& Corkin, 1995). Patients with a right occipital and posterior parietal cortical lesion displayed impaired performance on implicit tests of visual priming for words, but intact performance on explicit tests of recognition of words. In contrast, the reverse pattern was present for amnesic subjects with hippocampal damage. Furthermore, for patients with parietal lesions resulting in spatial neglect, there is a deficit in spatial repetition priming without a loss in short- term, working memory or recognition of spatial information (Ellis, Della Sala, \& Logie, 1996). Thus, it appears that perceptual memory based on implicit measures to assess long-term memory and short-term recognition memory based on explicit memory representations can operate independently of each other and can be processed by distinct neural regions.

In summary, data have been presented in support of the idea that the PPC supports a structural perceptual system in the form of a spatial cognitive map reflecting the operation of perceptual representations of relations among spatial features and landmarks as well as the operation of attentional processes to bind spatial features and landmarks and to selectively attend to the critical features that form spatial cognitive maps.

\section{REFERENCES}

Benton, A. L. (1969). Disorders of spatial orientation. In P. J. Vinken \& G. W. Bruyn (Eds.), Handbook of clinical neurology (Vol. 3). Amsterdam: North-Holland.

Boyd, M. G., \& Thomas, R. K. (1977). Posterior association cortex lesions in rats: Mazes, pattern discrimination, and reversal learning. Physiological Psychology, 5, 455- 461

Chen, L. L., Lin, L.-H., Barnes, C. A., \& MCNaughton, B. L. (1994). Head-direction cells in the rat posterior cortex: II. Contributions of visual and ideothetic information to the directional firing. Experimental Brain Research, 101, 24-34.

Chen, L. L., Lin, L.-H., Green, E. J., Barnes, C. A., \& McNaughton, B. L. (1994). Head-direction cells in the rat posterior cortex: I. Anatomical distribution and behavioral modulation. Experimental Brain Research, 101, 8-23.

Chen, L. L., \& Nakamura, K. (1998). Head-centered representation and spatial memory in rat posterior parietal cortex. Psychobiology, 26, 119-127.

Chiba, A. A., Jackson-Smith, P., \& Kesner, R. P. (1991). A double dissociation between implicit and explicit spatial memory following hippocampal and parietal cortex lesions. Society for Neuroscience Abstracts, 17, 131.

Cho, Y. H., \& KeSNeR, R. P. (1996). Involvement of entorhinal cortex or parietal cortex in long-term spatial discrimination memory in rats: Retrograde amnesia. Behavioral Neuroscience, 110, 436-442.

Cho, Y. H., Kesner, R. P., \& Brodale, S. (1995). Retrograde and anterograde amnesia for spatial discrimination in rats: Role of hippocampus, entorhinal cortex, and parietal cortex. Psychobiology, 23, 185-194.

CoRWIN, J. V., \& ReEP, R. L. (1998). Rodent posterior parietal cortex as a component of a cortical network mediating directed spatial attention. Psychobiology, 26, 87-102.

Crowne, D. P., Novotny, M. F., Maier, S. E., \& Vitols, R. (1992). Effects of unilateral parietal lesions on spatial localization in the rat. Behavioral Neuroscience, 106, 808-819.

Crowne, D. P., Richardson, C. M., \& Dawson, K. A. (1986). Parietal and frontal eye field neglect in the rat. Behavioural Brain Research, 22, 227-231.

Davis, B. K., \& McDaniel, W. F. (1993). Visual memory and visual spatial functions in the rat following parietal and temporal cortex injuries. Physiology \& Behavior, 53, 145-151.

DeCoteau, W. E., \& KeSNER, R. P. (1998). Effects of hippocampal and parietal cortex lesions on the processing of multiple-object scenes. Behavioral Neuroscience, 112, 68-82.

DERENZI, E. (1982). Disorders of space exploration and cognition. New York: Wiley.

DiMattia, B. V., \& Kesner, R. P. (1988a). Role of the posterior parietal association cortex in the processing of spatial event information. Behavioral Neuroscience, 102, 397-403.

DiMatTia, B. V., \& Kesner, R. P. (1988b). Spatial cognitive maps: 
Differential role of parietal cortex and hippocampal formation. Behavioral Neuroscience, 102, 471-480.

Ellis, A. X., Della Sala, S., \& Logie, R. H. (1996). The bailiwick of visuo-spatial working memory: Evidence from unilateral spatial neglect. Cognitive Brain Research, 3, 71-78.

Friedman-Hill, S. R., Robertson, L. C., \& Treisman, A. (1995). Parietal contributions to visual feature binding: Evidence from a patient with bilateral lesions. Science, 269, 853-855.

Gabrieli, J. D. E., Fleischman, D. A., Keane, M. M., Reminger, S. L., \& MoRrELL, F. (1995). Double dissociation between memory systems underlying explicit and implicit memory in the human brain. Psychological Science, 6, 76-82.

HABIB, M., \& SIRIGU, A. (1987). Pure topographical disorientation: A definition and anatomical basis. Cortex, 23, 73-85.

Heilman, K. M., Watson, R. T., \& Valenstein, E. (1993). Neglect and related disorders. In K. M. Heilman \& E. Valenstein (Eds.), Clinical neuropsychology (3rd ed., pp. 279-336). New York: Oxford University Press.

Jucker, M., Kametani, H., Bresnahan, E. L., \& Ingram, D. K. (1990). Parietal cortex lesions do not impair retention performance of rats in a 14-unit T-maze unless hippocampal damage is present. Physiology \& Behavior, 47, 207-212.

Keane, M. M., Gabrieli, J. D. E., Mapstone, H. C., Johnson, K. A., \& CORKIN, S. (1995). Double dissociation of memory capacities after bilateral occipital-lobe or medial temporal-lobe lesions. Brain, $118,1129-1148$.

KeSNER, R. P., FARNSwORTh, G., \& Kametani, H. (1992). Role of parietal cortex and hippocampus in representing spatial information Cerebral Cortex, 1, 367-373.

KING, V. R., \& CoRwIN, J. V. (1992). Spatial deficits and hemispheric asymmetries in the rat following unilateral and bilateral lesions of posterior parietal or medial agranular cortex. Behavioural Brain Research, 50, 53-68.

KING, V. R., \& CoRwIN, J. V. (1993). Comparison of hemi-inattention produced by unilateral lesions of posterior parietal cortex or medial agranular prefrontal cortex in rats: Neglect, extinction, and the role of stimulus distance. Behavioural Brain Research, 54, 117-131.

Kolb, B., Buhrmann, K., McDonald, R., \& Sutherland, R. J. (1994). Dissociation of the medial prefrontal, posterior parietal, and posterior temporal cortex for spatial navigation and recognition memory in the rat. Cerebral Cortex, 6, 664-680.

KOLB, B., \& CIOE, J. (1998). Absence of recovery or dendritic reorganization after neonatal posterior parietal lesions. Psychobiology, 26, 134-142.

KOLB, B., \& WALKEY, J. (1987). Behavioural and anatomical studies of the posterior parietal cortex in the rat. Behavioural Brain Research, 23, 127-145

LONG, J. M., \& KESNER, R. P. (1995). The effects of hippocampal and parietal cortex lesions on memory for an object/spatial location paired associate task in rats. Society for Neuroscience Abstracts, 21, 1215.

LONG, J. M., \& KESNER, R. P. (1996). The effects of dorsal versus ventral hippocampal, total hippocampal, and parietal cortex lesions on memory for allocentric distance in rats. Behavioral Neuroscience, $110,922-932$.

LONG, J. M., \& Kesner, R. P. (in press). The effects of hippocampal and parietal cortex lesions on memory for egocentric distance and spatial location information in rats. Behavioral Neuroscience.

LonG, J. M., Mellem, J. E., \& KeSNer, R. P. (1998). The effects of parietal cortex lesions on an object/spatial location paired-associate task in rats. Psychobiology, 26, 128-133.

MCDANIEL, W. F., \& SKEEL, R. L. (1993). Visual-spatial functions persist following temporal and posterior parietal cortex lesions in rat. Physiology \& Behavior, 53, 199-203.
MCDANIEL, W. F., \& WALL, T. T. (1988). Visuospatial functions in the rat following injuries to striate, peristriate, and parietal neocortical sites. Psychobiology, 16, 251-260.

MCDaniel, W. F., Williams, L. B., Attaway, C. M., \& Compton, D. M. (1998). Turn-signal utilization by rats with either unilateral or bilateral posterior parietal injuries. Psychobiology, 26, 143-152.

McNaughton, B. L., Chen, L. L., \& Marcus, E. J. (1991). "Dead reckoning," landmark learning, and the sense of direction: A neurophysiological and computational hypothesis. Journal of Cognitive Neuroscience, 3, 190-202.

McNaughton, B. L., Mizumori, S. J., Barnes, C. A., Leonard, B. J., MARQUIS, M., \& GREEN, E. J. (1994). Cortical representation of motion during unrestrained spatial navigation in the rat. Cerebral Cortex, 4, 27-39.

NakamURa, K., \& TAKarajima, A. (1996). Recognition of pattern position and shape by population vector in the spatial spreading associative neural network. IEEE International Conference on Evolutionary Computation (pp. 780-785).

PoSNER, M. I. (1980). Orienting of attention. Quarterly Journal of Experimental Psychology, 32, 3-25.

Posner, M. I., Walker, J. A., Friedrich, F. J., \& Rafal, R. D. (1984). Effects of parietal injury on covert orienting of attention. Journal of Neuroscience, 4, 1863-1874.

Rafal, R., \& Roberston, L. (1995). The neurology of visual attention. In M. Gazzaniga (Ed.), The cognitive neurosciences, (pp. 625648). Cambridge, MA: MIT Press.

Reer, R. L., Chandler, H. C., King, V., \& CoRwin, J. V. (1994). Rat posterior parietal cortex: Topography of cortico-cortical and thalamic connections. Experimental Brain Research, 100, 67-84.

Rosner, A. L., \& Mirtleman, G. (1996). Visuospatial attention in the rat and posterior parietal cortex lesions. Behavioural Brain Research, 79, 69-77.

Save, E., Poucet, B., Foreman, N., \& Buhot, M.-C. (1992). Object exploration and reactions to spatial and nonspatial changes in hooded rats following damage to parietal cortex or hippocampal formation. Behavioral Neuroscience, 106, 447-456.

Save, E., Poucer, B., Foreman, N., \& Thinus-Blanc, C. (1998). The contribution of the associative parietal cortex and hippocampus to spatial processing in rodents. Psychobiology, 26, 153-161.

SCHACTER, D. L. (1994). Priming and multiple memory systems: Perceptual mechanisms of implicit memory. In D. L. Schacter \& E. Tulving (Eds.), Memory systems 1994 (pp. 233-268). Cambridge, MA: MIT Press.

Spangler, E. L., Heller, B., Hengemihle, J., Muth, N. J., Jones, B. E., Garofalo, P., \& Ingram, D. K. (1994). Thrombosis of parietal, but not striate, cortex impairs acquisition of a 14-unit T-maze in the rat. Physiology \& Behavior, 56, 95-101.

Sutherland, R. J., Whishaw, I. Q., \& KolB, B. (1988). Contributions of cingulate cortex to two forms of spatial learning and memory. Journal of Neuroscience, 8, 1863-1872.

Thomas, R. K., \& WeIR, V. K. (1975). The effects of lesions in the frontal or posterior association cortex of rats on Maze III. Physiological Psychology, 3, 210-214.

UNGERLEIDER, L. G. (1995). Functional brain imaging studies of cortical mechanisms of memory. Science, 270, 769-775.

WARD, N. M., \& Brown, V. J. (1997). Deficits in response initiation, but not attention, following excitotoxic lesions of posterior parietal cortex in the rat. Brain Research, 775, 81-90.

(Manuscript received March 2, 1998; revision accepted for publication March 19, 1998.) 